\title{
Terahertz Imaging Patch Antenna for Cancer Diagnosis Applications
}

\author{
Kanmani Ruby E. D., Edwin Santhkumar W., Ponnusamy P.
}

\begin{abstract}
Cancer is the world second most common syndrome which causes death. In statistics nearly one of every four death occurs day-to-day, but it is remediable if detected earlier. The numerous imaging technologies exist for diagnosis with different constrains but those techniques provide either macroscopic or microscopic imaging. Histopathology of biopsy samples uses microscopic imaging methods to provide corporeal and functional information. In macroscopic level $X$-ray and MRI are used to provide images of living tissues and can achieve only much poorer resolution and specificity. Moreover it is harmful in terms of radiation and other causes To syndicate the macroscopic and microscopic imaging progresses, for delineating the precise margins of cancers is one of the prime mysterious complication in medical imaging.The $\mathrm{THz}$ tomography research does that syndication and achieves much high resolution with Non-ionizing radiation. The proposed nanomaterial based Microstrip antenna is pertinent to do $\mathrm{THz}-$ tomography with low cost and high resolution which can diagnosis and detection various cancers such as skin cancer, breast cancer, cervical cancer and colon cancer. In this paper, a terahertz imaging antenna was designed and analyzed using Ansys - HFSS v.14 simulation tool.
\end{abstract}

Keywords: Terahertz tomography, Cancer diagnosis, Nanotechnology, Antenna.

\section{INTRODUCTION}

In this digital world, the medical imaging field is drastically enhanced due to advanced biomedical technologies. Several pathological conditions are recognized and treated as earlier as possible. The novel methods proposed non-ionizing techniques as shown in figure 1, which will improve prediction of life critical diseases. It is supposed that nanotechnology is extremely going to inspire our everyday life and dramatically change the medical science world in $21^{\text {st }}$ century.This paper deliberate show nanotechnology can improve Terahertz (THz) imaging [1]. T $\mathrm{h}$ i s i s an emerging imaging modality for therapeutic applications. Terahertz radiation lies between the microwave and infrared band. It can also be called as $\mathrm{THz}$ waves, $\mathrm{THz}$ light, or T-rays. The regime is from $0.1 \mathrm{THz}$ to $10 \mathrm{THz}$ and is the most hunted research domain in physics, chemistry, biology, materials science and medicine. Although $\mathrm{THz}$ imaging has the potential to be used in many biomedical fields it is characterized by the use of non- ionizing radiation.

Revised Manuscript Received on February 19, 2020.

* Correspondence Author

Dr. E. D. Kanmani*, Professor, Department of Electronics and Commnication, Veltech University, Chennai, Tamil Nadu, India. E-mail: dredkanmaniruby@veltech.edu.in

Dr. W. Edwin Santhkumar, Department of Mechanical Engineering. SIT, Tumkur, Karnataka, India. E-mail: weskedwin@gmail.com

Dr. Ponnusamy P., Faculty, Department of Mechanical Engineering, Vellore Institute of Technology, Vellore, Tamil Nadu, India. E-mail: sreepons@gmail.com

(c) The Authors. Published by Blue Eyes Intelligence Engineering and Sciences Publication (BEIESP). This is an open access article under the CC BY-NC-ND license (http://creativecommons.org/licenses/by-nc-nd/4.0/)
If high resolution and efficient detection is required for terahertz tomography, it can be attained only by incorporating nanotechnology technology into it [11]. The terahertz frequency band is extremely sensitive to water content. All the tissues are made of water molecule about $70 \%$ and so this tomography method is well suited for various cell and tissue categorization [9]. In general, $\mathrm{H}_{2} \mathrm{O}$ molecules and all glacial liquids are engrossing the entire Terahertz frequency band. The significance of T-ray is its opacity in humid tissue which will permit both the enlargement of imaging used in vitro studies and in vivo imaging in transition and reflection categories [2]

The $\mathrm{THz}$ radiation can be penetrating through organic materials without any ionization. It categorizes the various materials by considering the water content and this will help to find Molecular recognition and protein folding. This method becomes peak research due to its specific physiognomies with interaction of molecules and tissue particularly in cancer spectra.

This work mainly focuses on the prospective of terahertz imaging and spectroscopy for cancer diagnosis. The conventional cancer detection method uses X-ray radiation for CT, MRI, and PET. The problem with X-ray is, it's radiation are about (0.12 to 120$) \mathrm{keV}$ so it hurts biological tissues but the proposed tomography of $\mathrm{THz}$ range is that it only emits the energy of around 4.14 $\mathrm{meV}$ (this is thousand times smaller energy then $\mathrm{X}$-ray). Hence this T-ray will not make any ionization deathtrap to biological tissues [8]. The size of the conventional to mographic system is very much bigger and maintaining such devices requires sophisticated arrangements such as X-ray, CT, PET, and MRI required bizarre room and technical assistance [4]. This leads diagnosis process much costly so the poor people could not undertake this medical assistance though they need. Here this proposed method is based on simply an antenna with $\mathrm{THz}$ sensitivity and some signal processing units, so this ensures detection of cancers with minimum cost because this device need not required any such sophisticate room and technical issues and even or people can also undergo this diagnosis test.

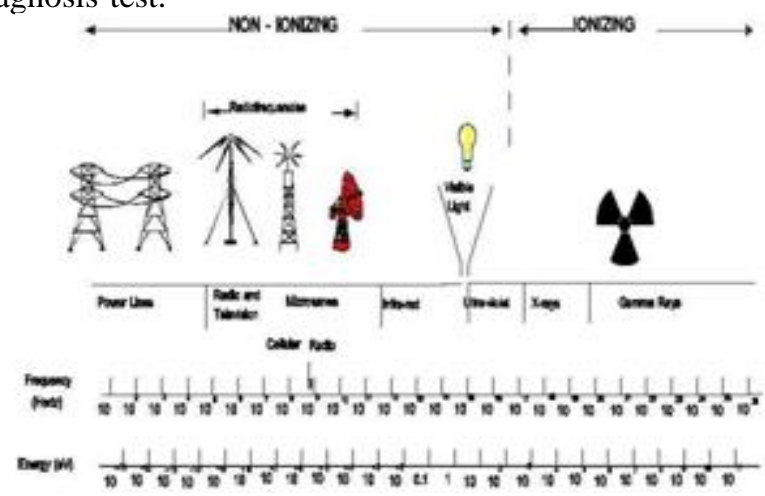

Fig 1: The Electromagnetic Spectrum 


\section{BACK GROUND WORK}

In existing technology several non-invasive techniques are used with exclusive benefit and shortcomings. The proposed work gives solution to overcome those shortcomings by several aspects. A wide variety of Non-invasive techniques are used for cancer detection such as Ultrasound, MRI, PET scans, CT scans, X-rays and though these are non-invasive, their tribulations due to their characteristics. More over cancer diagnosis needs timely detection but those conventional methods are not exactly attaining it [10]. Some specific detection techniques are Mammography, Sigmoidoscopy, Virtual Colonoscopy, Pap smears, Prostate specific antigen that are recently used can detect specific cancer a bit earlier than the above methods but are not comfortable and also has certain side effects. Because these techniques contribute to physical connectivity and will make the patient feel hesitant during the process. But the proposed system does not require any physical connectivity or insideview camera and so on. The proposed antenna simply passes the T-ray to the object and diagnoses many cancers at initial stage itself as it is considering the root of the syndrome cell.

Some of the popular diagnoses methods and there shortcoming are discussed below,

Mammography method is used to diagnose breast cancer. Here X-ray is used, with low magnitude to create an image of a cancer cell. In this method it will not produce exact anomalies because this imaging technique misses to detect and/or fail to detect the cancer cell. So it insinuations to additional testing creates anxiety in patient. It also exposes radiation hazards but lesser then conventional X-ray. The person who have undergone mammography, need to place the respective organs in between two plastic plates.

Colonoscopy method is use to detect colon cancer by using colonoscope, commonly called scope which has a tiny camera attached to view inside the entire patient large intestine. This thin tube or short camera is to stare reddened tissue, unusual growths, and ulcers. But this method leads to Bleeding, diverticulitis, perforation and severe abdominal pains which are the most common complications from colonoscopy. The gastroenterologist can treat bleeding that occurs during the period of test but in some case the patient may have delayed bleeding after some few weeks.

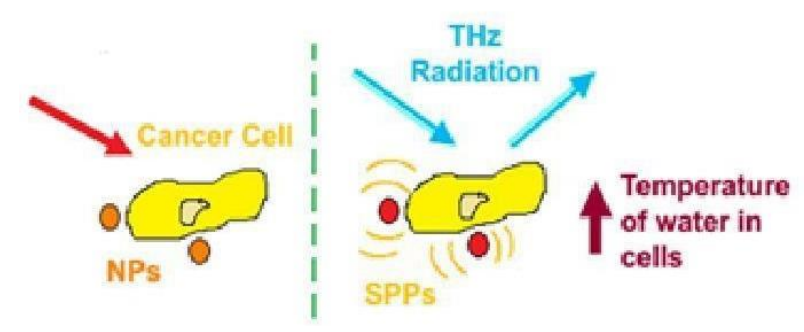

Fig 2: The Hyperthermia Effect

The microstrip line feed is used between rectangular patch Mostly $\mathrm{Gd}_{2} \mathrm{O}_{3}$ (gadolinium oxide) NPs are used as terahertz imaging contrast agent. It has better interaction in THz range.

Moreover, the $\mathrm{Gd}_{2} \mathrm{O}_{3}$ nano $\mathrm{S}$ particles are commonly used in MRI so it can combine benefit of both MRI and $\mathrm{THz}$ imaging modalities. Apart from the previously mentioned advantages of diagnosis it also used in drug delivery monitoring [2].
Sigmoidoscopy consist of small flexible scope which is used to view the colon lower part and their screening method can help to view the last portion of colon, so that it cannot view the complete zone of cancer cell in colon. Likewise the existing methods obligate so many problems to the patient in both physical and mental aspects, but by using proposed system all the shortcomings are elucidated also it gives very high resolution imaging and effective detection. These above literature are taken from various medical center journals referred in [7, 8, and 9].

The proposed tomography techniques have advantage over commercial methods which is given below,

- $\quad$ It emits Non-ionizing radiation harmless for biological tissues.It senses or detects the cancer symptoms as earlier because it focuses on biological molecules' characteristics by hyperthermia effect.

- In this method patient feel comfortable due to its contact free tomography.

- $\quad$ Compare to other frequencies the terahertz preserve high spatial resolution.

- In this frequency range the contribution of Rayleigh scattering is very small compared to X-ray MRI.

This spectroscopy information provides both morphological and biochemical features.

\section{TERAHERTZ IMAGING MODALITIES}

The main principle of THz-imaging antenna is can detect the hyperthermia effect. The benefit of this method is non- connectivity diagnosis system. The existence of cancer often causes augmented blood circulation and water content in the affected tissues. The terahertz tomography works with this benefit and in order to attain more clarity of imaging result the nanotechnology is used.

The nanoparticle is used as contrast agents to enhance sensitivity in $\mathrm{THz}$ signals. The contrast agent is injected to the patient before imaging process starts shown in figure 1. After the NPs are injected the cancer cells start to react differently because Surface Plasma Polaritons (SPPs) occurs. Due to SPPs, the cancer cells water temperature enlarged so that it could be detected by the THz antenna [12, 13].

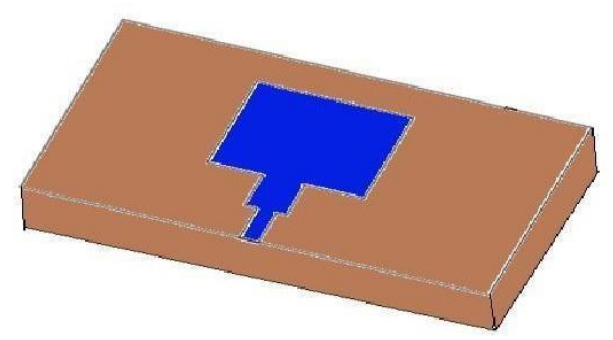

Fig 3: Prototype of the Proposed THz Antenna

\section{PROPOSED IMAGING ANTENNA}

The microstrip patch antenna is considered to be an important device because of its compatibility for miniaturization in terahertz frequency regime. The $\mathrm{THz}$ antenna affords secured data transfer and ultra widebroadband in the future wireless communication systems. 
The proposed microstrip patch antenna consists of a Graphene nano ribbon material as radiated patch and polyimide materiel as dielectric substrate. The dimension of the substrate and ground plate of the proposed antenna is $210 \mu \mathrm{m} \times 434 \mu \mathrm{m} \times 20 \mu \mathrm{m}$, respectively as shown in figure 2. The thickness of the dielectric substrate is in the range of $0.003 \lambda_{0} \leq \mathrm{t} \leq 0.05 \lambda_{0}$ and patch thickness is much lesserthen $\lambda_{0}$; where $t$ is substrate thickness and $\lambda_{0}$ is free space wave length. The designed values are calculated using formulas and design constrains discussed in [3].

The reason behind the elected patch material as Graphene material which has promising electrical conductivity, electromag netism and electro mechanical properties for forthcoming generation of molecular electronics. The flexible dielectric material is polyimide $\varepsilon \mathrm{r}=3.5$ with $\tan \delta=$ 0.008 is used as substrate with a dimension of $89.23 \mu \mathrm{m} \times$ $135.96 \mu \mathrm{m}$.

In order to improve radiation efficiency, low dieletric permittivity material is needed.[3].The fringing effects are the main contributing factor in electromagnetic radiation. In order to reduce that effect, the patch length and width should be $L / W>>1$ (here attained as 0.67 ). This ensures the lowest fringing effect [3] and the substrate with small values of dielectric permittivity is preferred, to enhances the fringing fields.

and coaxial line for impedance transition. The antenna is fed by a $50 \Omega$ microstrip transmission line.The width of the microstrip line is optimized to match the antenna impedance with negligible mismatch loss with a dimension of $45 \mu \mathrm{m}$ $\times 20 \mu \mathrm{m}$ and $18 \mu \mathrm{m} \times 4 \mu \mathrm{m}$, respectively designed by considering various conditions in [3] and obtain the resultant prototype as shown in Fig 2.

\section{RESULTS AND DISCUSSION}

Using Ansys HFSS v.14 simulation tool ,the antenna is designed and its radiation characteristics are examined by finite element method. The various analyzed parameters are given below,

\section{A. Return Loss}

The return loss $\left(\mathrm{S}_{11}\right)$ of the proposed patch antenna is shown in Fig 4. The return loss is obtained as $-30 \mathrm{~dB}$ in resonating frequency range from 0.725 to 0.775 in terms of Terahertz.

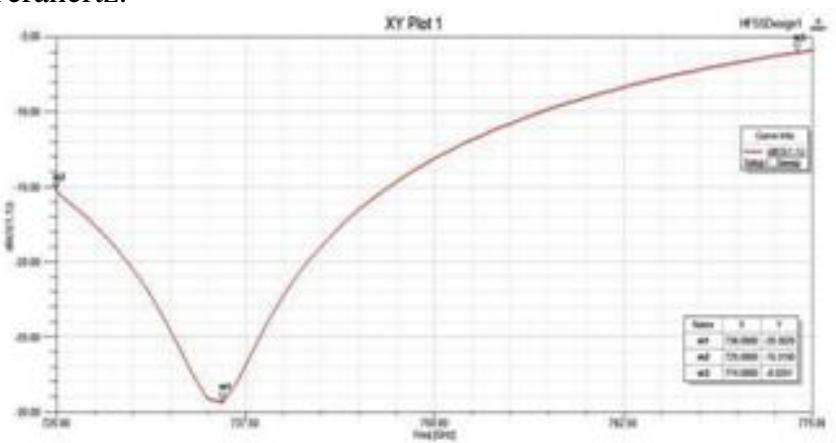

Fig 4: Return Loss (S11) Vs. Frequency

\section{B. Current Distribution}

Current distribution of the antenna shows how the current is distributed in the antenna surface in feed line and in other edges.

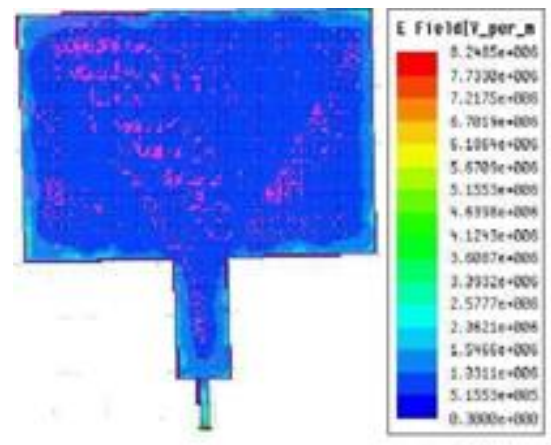

Fig 5: Current Distribution of Proposed Antenna

Fig 5 represents the simulated current distributions on the antenna surface. In the solution frequency, the current density becomes more concentrated along the edges. The above result shows that the distributed power is very low (indicated as blue in colour).

\section{Radiation Pattern}

It is the display of the antenna radiation characteristics in the Far-Field region between radial distance and frequency. Figure 6 shows the radiation pattern at angel $\phi$ and $\theta$ correspondingly at solution frequency in 3D plot with the far- field radiation characteristics of an antenna with a function of the spatial coordinates with better frontto back lobe ratio is achieved.

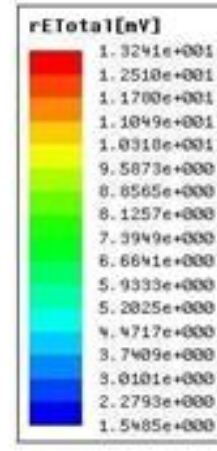

VSWR

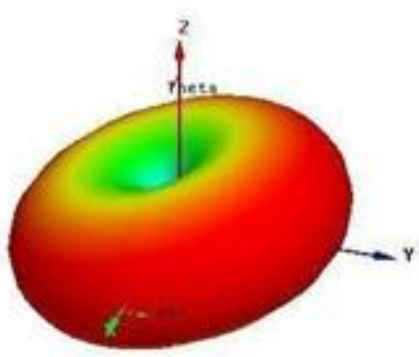

Fig 6: 3D radiation pattern
To achieve maximum energy transfer between sources to the antenna, impedance should be matched. If the impedance is not matched properly then the energy is not transferred. It will return back to the source. This makes unnecessary standing waves and in order to avoid such case here the proposed antenna is engineered. Ideally the VSWR need to be low (between the range of 1-2) from Fig7, it is clearly show that the proposed antenna attains it. 


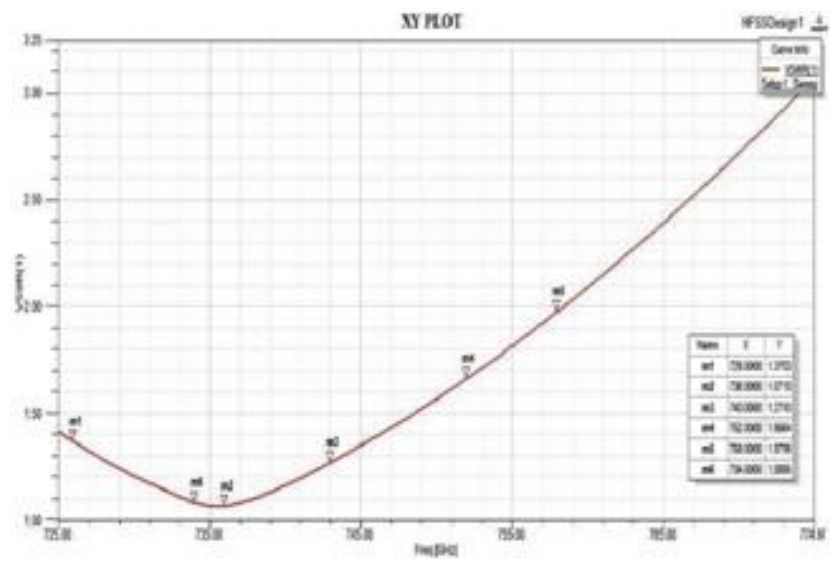

Fig 7: Frequency vs VSWR

\section{SRA Measurement}

The specific rate absorption test is used to test the energy absorption rate of the human tissues in the presence of electromagnetic radiation. It refers the power absorbed per mass of tissue and it has units of watts per kilogram.

$$
S A R=\int \frac{\sigma(x)|E(x)|^{2}}{\rho(x)} d x
$$

Where, $\sigma(x)$

$E(x)$

$\rho(x)$

is the samples electrical conductivity is the RMS electric field is the sample density

The SAR simulation result from Ansoft HFSS shows the SAR rate is $3.8 \mathrm{~W} / \mathrm{kg}$. Generally for whole human body the SAR typical rate is $4 \mathrm{~W} / \mathrm{kg}$ [14].

\section{CONCLUSION}

Cancer is a disease which is difficult to diagnose timely, but by using Terahertz radiation tomography the cancer affected cell can be effectively detected better success rate than the existing imaging methods. The conventional diagnose techniques causes' radiation and also have many difficulties as reviewed in section II. But here the proposed antenna makes detection as easier and earlier since the $\mathrm{THz}$ radiation is sensitive to hyperthermia effect of tissues.

The proposed antenna is very smaller in size in terms of new thundered micrometer and hence consumes very low power of $3.58 \mathrm{mV}$ and it gives high resolution image compared with all other traditional cancer detection method and also reviewed in section III. From the result, the SAR value is found to be $3.8 \mathrm{~W} / \mathrm{kg}$ which is the desirable for human being which can withstand a maximum value of $4 \mathrm{~W} / \mathrm{kg}$ and hence it is inferred that the designed antenna is well suitable for medical imaging applications and ensures hazards free radiation. In future the antenna size is to be reduced to nano range and further to combine nano antennas to form micro size array antenna with improved clarity and much reduced power.

\section{REFERENCES}

1. Andreas Stylianou, Michael A Talias, "Nanotechnology Supported Thz Medical Imaging” F1000 Research-2014.

2. Calvin Yu, Shuting Fan, Yiwen Sun, "The Potential Of Terahertz Imaging For Cancer Diagnosis: A Review Of Investigation To Date" Quantitative Imaging In Medical And Surgery, Isdn:2223-4292; Vol2, 2012.

3. Constantine A. Balanis, A Hand Book On "Antenna Theory: Analysis and Design” (3edition) John Wiley\& Sons, 2005.
4. Dranthonyb.Wolbarst, Drwilliamr.Hendee, "Evolving And

5. Experimental Technologies In Medical Imaging" Radialogy: Vol238.

6. Houriaboulaiz, Pablo J. Alvarez, "Nanomedicine: Application Areas and Development Prospects" International Journal Of Molecular Sciences, Issn 1422-0067, Doi: 10.3390

7. /Ijms12053303.

8. Amenabar ,F. Lopez \&A. Mendikute, "In Introductory Review to Thz Non-Destructive Testing Of Composite Matter", J Infrared Milliterahz Waves (2013) 34:152-169, Doi: 10.1007/S10762-0129949-Z.

9. Journal From, "Penn State Hersheymiltons.Hershey Medical Center", Adam Multimedia Encyclopedia.

10. Journal From, "Energy Based Medical Technologies and Therapies".

11. Journal From "University Of Maryland Medical Center".

12. Partricke.Young, Craigm.Womeldorph "Early Detection

13. of Colorectal Cancer Recurrence In Patients Undergoing Surgery With Curative Intent: Current Status And Challenges" Journal Of Cancer- 2014, Doi:10.7150/Jca.7988.

14. Seungjae Oh \&Yong-Min Huh "Cancer Diagnosis By Terahertz Molecular Imaging Technique" J Infrared Milliterahz Waves (2012) 33:74-81, Doi: 10.1007/S10762-011-9847-9.

15. U.S. Department Of Health And Human Services "National Digestive Diseases Information Clearing House".

16. Yiwen Sun, Mingyiusy "A Promising Diagnostic Method:

17. Terahertz Pulsed Imaging And Spectroscopy" World Journal of Radiology, 2011; Vol: 55-65,Doi:10.4329/Wjr.V3.I3.55.

18. Srithar A, Dr.E.D.Kanmani Ruby, "Planar Inverted Multiband Slotted Patch Antenna For Rfid Application",Journal Of Asdf, India, Isbn: 978-81 -929742-1-7, Pp.88-94, Jan 2015.

19. Meidyawati Virginia Hidayat and Catur Apriono Simulation of terahertz imaging using microstrip linear array antenna for breast cancer detection AIP Conference Proceedings 2092, 020020 (2019).

\section{AUTHORS PROFILE}

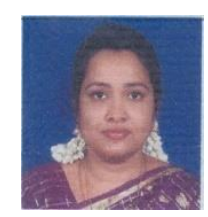

Dr. E. D. Kanmani, Ruby, completed her graduation in the year 1996 ,in the Department of Electronics and Communication Engineering.Her masters degree is in Applied Electronics in the year 2005.She finished her doctoral degree in Information and Communication in the year 2013.During her career she has published more than 20 papers in reputed Journals. She is life member of ISTE and IETE. Her area of interest include Communication and Networking.She is currently working as Professor in the Department of Electronics and Commnication at Veltech University, Chennai.

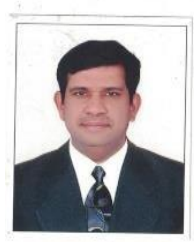

Dr. W. Edwin Santhkumar, completed his bachelors from SIT,Tumkur in the Department of Mechanical Engineering. His Masters degree is in Engineering Design in the year 2005. He finished his PhD in the year 2017.During his career he has published more than 10 papers in reputed Journals. His area of interest includes manufacturing design and his resear ch work is on multidisciplinary measurement systems.

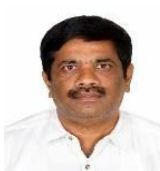

Dr. Ponnusamy P., completed his Bachelors in Engineering from the Coimbatore Institute of Technology, Coimbatore, Bharathiar University and went on pursue his M.Tech. (Energy Technology) from Pondicherry EngineeringCollege,

Pondicherry, Pondicherry University and his $\mathrm{PhD}$ in Anna University, Chennai. He has over 25 years of rich

\& extensive experience in Teaching. Currently he is associated with Vellore Institute of Technology ,as Faculty in School of Mechanical Engineering. He has published more than 25 research papers in Journals and Conference, written a book on Total Quality Management, has organized more than 10 Faculty Development Programmes . 